\title{
THE ELECTROMAGNETIC FIELD PRODUCED BY A HELIX*
}

\author{
BY \\ R. S. PHILLIPS \\ University of Southern California
}

1. Introduction. In this paper ${ }^{1}$ we are concerned with finding the electromagnetic field produced by an infinitely-thin perfectly-conducting helix subject to a monochromatic excitation. Because of the use of the helix in an ultra-high frequency amplifier known as the traveling-wave tube [2], there has been in recent years considerable interest in this problem. Earlier papers dealing with the field due to a helix have been based on a model consisting of a circular cylinder which conducts only in a given helical direction. ${ }^{2}$ [1], [2], [3]. The symmetry of this idealization corresponds to that of cylindrical coordinates so that such a model yields to the usual separation of variables technique. Our approach is quite different.

We start off by assuming that the effect of a monochromatic electromagnetic field would be to induce a sinusoidal electric current on the helical conductor; and that this current travels along the helix with a real propagation constant $\beta$ in the axial direction. ${ }^{3}$ The electromagnetic field accompanying such a current may then be represented by means of the retarded vector potential $\Pi$ as $[5, p .165]$

$$
\Pi(x, y, z, t)=A \int([i] / r) d s,
$$

where [i] is the current vector at a point $(\xi, \eta, \zeta)$ of the helix at time $(t-r / c), c$ being the free space wave velocity, $r=\left[(x-\xi)^{2}+(y-\eta)^{2}+(z-\zeta)^{2}\right]^{1 / 2}, s$ the arc length along the helix, and $A$ a scalar factor.

It follows that

$$
E=\nabla(\nabla \cdot \Pi)+k^{2} \Pi, \quad H=-i \omega \epsilon \nabla \times \Pi,
$$

where $k^{2}=\epsilon \mu \omega^{2}, \omega$ being the radial frequency of the field. We then attempt to determine $\beta$ so as to make the electric field along the prefectly conducting helix "essentially" zero, and thus validate our initial assumption. It will turn out that the $\beta$ so determined corresponds to a phase velocity along the helix itself equal to $c$.

This then is our program. The principal difficulty lies in the singular nature of the potential function $\Pi$. In Sec. 2 we show that the integral defining $\Pi$ converges; and in addition we obtain an evaluation for $\Pi$ in terms of Bessel functions. In fact we establish

$$
\int_{-\infty}^{\infty} e^{i \beta \zeta}\left(e^{i k R} / R\right) d \zeta=\sum_{m=-\infty}^{\infty} 4 \pi e^{i n \varphi} I_{n}\left(\Gamma_{n} a\right) K_{n}\left(\Gamma_{n} \rho\right), \quad(\rho>a)
$$

\footnotetext{
*Received June 20, 1949.

1The work on this paper was done under funds granted to New York University by the Air Force Cambridge Research Laboratories, under contract W28-097-ac-170.

${ }^{2}$ By helical direction we mean the direction of a vector field which in cylindrical coordinates has the components $v_{\rho}=0, v_{\varphi}=a, v_{z}=\alpha$.

${ }^{3}$ That is, the amplitude varies as $\cos (\beta z-\omega t)$ where $z$ is measured in the axial direction and $\omega$ is the radial frequency.
} 
where

$$
R=\left[\zeta^{2}+a^{2}+\rho^{2}-2 a \rho \cos (\varphi-\zeta / \alpha)\right]^{1 / 2}
$$

and

$$
\Gamma_{n}=\left[(\beta-n / \alpha)^{2}-k^{2}\right]^{1 / 2} .
$$

Here, $I_{n}$ and $K_{n}$ are modified Bessel and Hankel functions of $n$th order. If for a particular $n, \Gamma_{n}$ is imaginary, then the corresponding term in (1.3) is to be replaced by $2 \pi^{2} i e^{i_{n} \varphi} J_{n}\left(\gamma_{n} a\right) H_{n}^{(1)}\left(\gamma_{n} \rho\right)$, where $\gamma_{n}=\left[k^{2}-(\beta-n / \alpha)^{2}\right]^{1 / 2}$.

In Sec. 3 we prove that $I I$ may be differentiated either by differentiating under the integral sign or by differentiating its series expansion termwise. We then show that $\Pi$ satisfies the homogeneous wave equation at all points not on the helix. Also in Sec. 3 explicit expressions for the electromagnetic field are obtained. Finally in Sec. 4 the propagation constant $\beta$ is determined so that the boundary conditions for the helix are satisfied. Since the electric field is singular on the wire, $\beta$ is determined so that the ratio of the electric field in the helical direction for the proper $\beta$ to that of the electric field in the helical direction for any other $\beta$ approaches zero as the helix is approached radially.

In cylindrical coordinates, the equation for the helix is

$$
\rho=a, \quad z=\alpha \varphi .
$$

The assumed current along the helix is the real part of

$$
\begin{aligned}
& i_{\rho}=0 \\
& i_{\varphi}=I a\left[a^{2}+\alpha^{2}\right]^{-1 / 2} \exp [i(\beta z-\omega t)], \\
& i_{z}=I \alpha\left[a^{2}+\alpha^{2}\right]^{-1 / 2} \exp [i(\beta z-\omega t)] .
\end{aligned}
$$

The retarded potential at the point $(\rho, \varphi, z)$ and the time $t$ is the real part of

$$
\begin{aligned}
& \Pi_{\rho}=a F \int_{-\infty}^{\infty} r^{-1} \exp (i \beta \zeta+i k r) \sin (\varphi-\zeta / \alpha) d \zeta, \\
& \Pi_{\varphi}=a F \int_{-\infty}^{\infty} r^{-1} \exp (i \beta \zeta+i k r) \cos (\varphi-\zeta / \alpha) d \zeta, \\
& \Pi_{z}=\alpha F \int_{-\infty}^{\infty} r^{-1} \exp (i \beta \zeta+i k r) d \zeta
\end{aligned}
$$

where

$$
r=\left[(z-\zeta)^{2}+a^{2}+\rho^{2}-2 a \rho \cos (\varphi-\zeta / \alpha)\right]^{1 / 2}
$$

and

$$
F=-I e^{-i \omega t} / 4 \pi i \omega \epsilon \alpha .
$$

If we replace sines and cosines by their exponential representations, we see that all of the integrals in (1.6) are essential of the type found in $\Pi_{s}$. The notation of the next section will be somewhat simplified if we make a change of variables $\zeta^{\prime}=\zeta-z$ in these integrals. Then 


$$
\int_{-\infty}^{\infty} e^{i \beta \zeta}\left(e^{i k r} / r\right) d \zeta=e^{i \beta z} \int_{-\infty}^{\infty} e^{i \beta \zeta^{\prime}}\left(e^{i k r^{\prime}} / r^{\prime}\right) d \zeta^{\prime}
$$

where

$$
r^{\prime}=\left[\zeta^{\prime 2}+a^{2}+\rho^{2}-2 a \rho \cos \left[(\varphi-z / \alpha)-\zeta^{\prime} / \alpha\right]\right]^{1 / 2} .
$$

2. Evaluation of the integral. In this section we shall evaluate the integral

$$
I=\int_{-\infty}^{\infty} e^{i \beta \zeta}\left(e^{i k R} / R\right) d \zeta
$$

where

$$
R=\left[\zeta^{2}+a^{2}+\rho^{2}-2 a \rho \cos (\varphi-\zeta / \alpha)\right]^{1 / 2} .
$$

We restrict our consideration to real $\beta$ since the integral will clearly not converge for complex $\beta$.

The usual method of handling improper integrals, namely to transform the path of integration, is not suitable here because $R$ has an infinite set of branch points. These are the solutions of the transcendental equation $R^{2}=0$. The device which we shall use in evaluating (2.1) consists of replacing the integral along the helix by a double integral over the enveloping cylinder of the helix; this leads to a Bessel function solution.

We define

$$
I(L, r)=\int_{-L}^{L} d \zeta \int_{0}^{2 \pi} d \sigma e^{i \beta \zeta}\left(e^{i k \Re} / \Re\right) P(r, \sigma-\zeta / \alpha),
$$

where $P(r, \sigma)$ is the Poisson kernel $[7$, p. 51]

$$
P(r, \sigma)=\frac{1}{2 \pi} \frac{1-r^{2}}{1+r^{2}-2 r \cos \sigma}=\frac{1}{2 \pi} \sum_{n=-\infty}^{\infty} r^{|n|} e^{i n \sigma}
$$

and

$$
\Re=\left[\zeta^{2}+a^{2}+\rho^{2}-2 a \rho \cos (\varphi-\sigma)\right]^{1 / 2}
$$

For $0 \leq r<1, L<\infty, \rho>a$, the double integral is absolutely integrable and hence the order of integration may be interchanged.

By well known properties of the Poisson kernel

$$
\lim _{r \rightarrow 1-} \int_{0}^{2 \pi} d \sigma e^{i \beta \zeta}\left(e^{i k \Re} / \Re\right) P(r, \sigma-\zeta / \alpha)=e^{i \beta \zeta}\left(e^{i k R} / R\right)
$$

uniformly for $-L \leq \zeta \leq L$. Hence

$$
I=\lim _{L \rightarrow \infty} \lim _{r \rightarrow 1-} I(L, r)
$$

if this limit exists.

We wish to show not only that the limit of (2.4) exists but that the order of this double limit may be interchanged. In order to accomplish this we shall prove
a) $\lim _{L \rightarrow \infty} I(L, r) \quad$ exists for each $r(0 \leq r<1)$
b) $\lim _{r \rightarrow 1-} I(L, r) \quad$ exists uniformly in $L$ for $(0<L<\infty)$. 
Since the Fourier expansion of $P(r, \sigma)$ converges uniformly in $\sigma$ for each $r<1$, it follows that we can interchange the order of integration and summation and so express $I(L, r)$ as

$$
I(L, r)=\sum_{n=-\infty}^{\infty} r^{|n|} \int_{-L}^{L} d \zeta \int_{0}^{2 \pi} d \sigma \Re^{-1} \exp (i \beta \zeta+i k \Re) \exp [\operatorname{in}(\sigma-\zeta / \alpha)] .
$$

In order to establish (a) we first examine the individual terms of this sum. For the case $(\beta-n / \alpha)>k$, we make the substitution

$$
\begin{aligned}
\zeta & =\left[a^{2}+\rho^{2}-2 a \rho \cos (\varphi-\sigma)\right]^{1 / 2} \sinh (\psi-\delta), \\
\Re & =\left[a^{2}+\rho^{2}-2 a \rho \cos (\varphi-\sigma)\right]^{1 / 2} \cosh (\psi-\delta),
\end{aligned}
$$

where

$$
\begin{aligned}
& \cosh \delta=(\beta-n / \alpha) /\left[(\beta-n / \alpha)^{2}-k^{2}\right]^{1 / 2}, \\
& \sinh \delta=k /\left[(\beta-n / \alpha)^{2}-k^{2}\right]^{1 / 2} .
\end{aligned}
$$

Then

$$
(\beta-n / \alpha) \zeta+k \Re=\Phi \sinh \psi
$$

where we have set

$$
\Phi=\left[a^{2}+\rho^{2}-2 a \rho \cos (\varphi-\sigma)\right]^{1 / 2}\left[(\beta-n / \alpha)^{2}-k^{2}\right]^{1 / 2} .
$$

Hence,

$$
\int_{-L}^{L} d \zeta\left(e^{i k \Re} / \Re\right) \dot{\exp }[i(\beta-n / \alpha) \zeta]=\int_{-l_{1}}^{l_{2}} d \psi \exp (i \Phi \sinh \psi),
$$

where $l_{1}$ and $l_{2}$ are defined by

$$
\begin{aligned}
& L=\left[a^{2}+\rho^{2}-2 a \rho \cos (\varphi-\sigma)\right]^{1 / 2} \sinh \left(l_{1}+\delta\right), \\
& L=\left[a^{2}+\rho^{2}-2 a \rho \cos (\varphi-\sigma)\right]^{1 / 2} \sinh \left(l_{2}-\delta\right),
\end{aligned}
$$

$\delta$ being defined as above. We now transform the path of this integral to the line $\psi=i \pi / 2$. Then because of the fact that the integral along the line joining $(l, 0)$ and $(l, i \pi / 2)$ goes to zero as $l \rightarrow \pm \infty$, we get

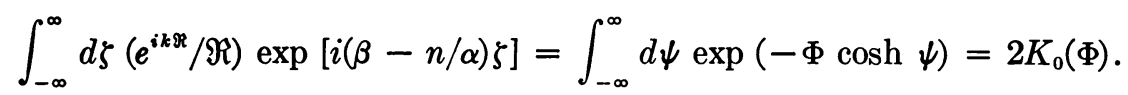

The latter equality can be found in [6, p. 182, (7)].

It is clear from the above argument that

$$
2 K_{0}(\Phi)-\int_{-L}^{L} d \zeta\left(e^{i k \Re} / \Re\right) \exp [i(\beta-n / \alpha) \zeta]=\int_{P} d \psi \exp (i \Phi \sinh \psi),
$$

where $P$ is the sum of the two paths indicated on Fig. 1 . 
For $\rho>a$, the integral

$$
\int_{P} d \psi \exp (i \Phi \sinh \psi)
$$

converges to zero uniformly in $\sigma$ as $l_{1}, l_{2} \rightarrow \infty$. This incidentally shows that the order of integration in

$$
\int_{0}^{2 \pi} d \sigma \int_{-\infty}^{\infty} d \zeta e^{i \beta \zeta}\left(e^{i k \Re} / \Re\right) \exp [i n(\sigma-\zeta / \alpha)
$$

may be interchanged. We can, however, easily obtain an upper bound in the value of this integral over $P$. In fact the integral from $(l, 0)$ to $(l, \pi i / 2)$ is bounded by $2 /(\Phi \cosh l)$;

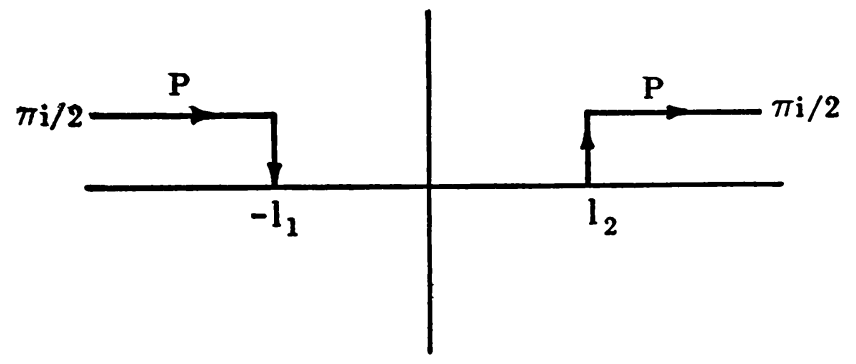

Fig. 1

whereas the integral from $(l, \pi i / 2)$ to $(\infty, \pi i / 2)$ is bounded by $e^{-\Phi l} / \Phi$. Now for sufficiently large $|n|$, fixed $\beta$, and fixed $\rho>a, \Phi \geq c|n|$. Hence in this case

$\left|\int_{0}^{2 \pi} d \sigma K_{0}(\Phi) e^{i n \sigma}-\int_{-L}^{L} d \zeta \int_{0}^{2 \pi} d \sigma e^{i \beta \zeta}\left(e^{i k \Re} / \Re\right) \exp [i n(\sigma-\zeta / \alpha)]\right|<\frac{\bar{c}}{|n|}$.

A similar argument shows that (2.7) and (2.8) hold equally well for $(\beta-n / 2)<-k$.

For the case $(\beta-n / 2)^{2}<k^{2}$, let

$$
\begin{aligned}
\zeta & =\left[a^{2}+\rho^{2}-2 a \rho \cos (\varphi-\sigma)\right]^{1 / 2} \sinh \left(\psi-\delta^{\prime}\right), \\
\Re & =\left[a^{2}+\rho^{2}-2 a \rho \cos (\varphi-\sigma)\right]^{1 / 2} \cosh \left(\psi-\delta^{\prime}\right),
\end{aligned}
$$

where now

$$
\begin{aligned}
& \sinh \delta^{\prime}=(\beta-n / \alpha) /\left[k^{2}-(\beta-n / \alpha)^{2}\right]^{1 / 2} \\
& \cosh \delta^{\prime}=k /\left[k^{2}-(\beta-n / \alpha)^{2}\right]^{1 / 2}
\end{aligned}
$$

For

$$
\Phi^{\prime}{ }^{\prime}=\left[a^{2}+\rho^{2}-2 a \rho \cos (\varphi-\sigma)\right]^{1 / 2}\left[k^{2}-(\beta-n / \alpha)^{2}\right]^{1 / 2}
$$

we have, then,

$$
(\beta-n / \alpha) \zeta+k \Re=\Phi^{\prime} \cosh \psi
$$


so that

$$
\int_{-L}^{L} d \zeta\left(e^{i k \Re} / \Re\right) \exp [i(\beta-n / \alpha) \zeta]=\int_{-l_{1}^{\prime}}^{l_{2}^{\prime}} d \psi \exp \left(i \Phi^{\prime} \cosh \psi\right) .
$$

The difference between this integral and the integral from $(-\infty-i \pi / 2)$ to $(-i \pi / 2)$ to $(i \pi / 2)$ to $(\infty+i \pi / 2)$ is the integral over the path $P^{\prime}$ shown in Fig. 2.

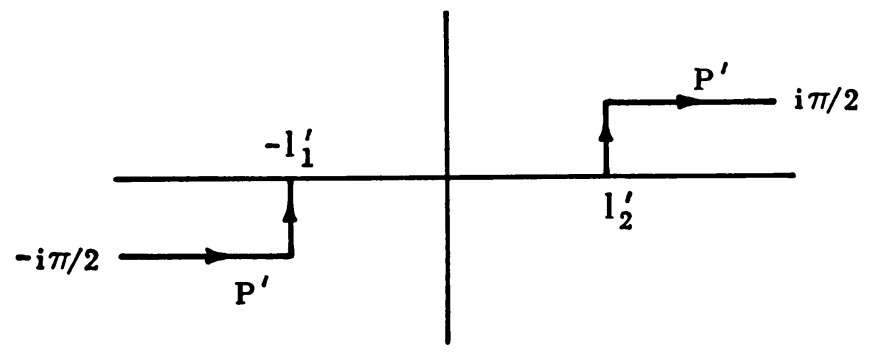

Fig. 2

For $\rho>a$, the integral $\int_{P}, d \psi \exp \left(i \Phi^{\prime} \cosh \psi\right)$ converges to zero uniformly in $\sigma$ as $l_{1}$, $l_{2} \rightarrow \infty$. Hence

$$
\begin{aligned}
\int_{-\infty}^{\infty} d \zeta\left(e^{i k \Re} / \Re\right) \exp [i(\beta-n / \alpha) \zeta] & =\int_{-\infty-i \pi / 2}^{\infty+i \pi / 2} d \psi \exp \left(i \Phi^{\prime} \cosh ^{\top} \psi\right) \\
& =\pi i H_{0}^{(1)}\left(\Phi^{\prime}\right)
\end{aligned}
$$

(see $[6$, p. 180, (8)]) and

$$
\int_{-\infty}^{\infty} d \zeta \int_{0}^{2 \pi} d \sigma e^{i \beta \zeta}\left(e^{i k \Re} / \Re\right) \exp [i n(\sigma-\zeta / \alpha)]=\int_{0}^{2 \pi} d \sigma H_{0}^{(1)}\left(\Phi^{\prime}\right) e^{i n \sigma} .
$$

Now by the addition theorem for Bessel functions [6, p. 361] for $\rho>a$

$$
\begin{aligned}
H_{0}^{(1)}\left(x\left[a^{2}+\rho^{2}-2 a \rho \cos \theta\right]^{1 / 2}\right) & =\sum_{n=-\infty}^{\infty} J_{m}(x a) H_{m}^{(1)}(x \rho) e^{i m \theta}, \\
K_{0}\left(x\left[a^{2}+\rho^{2}-2 a \rho \cos \theta\right]^{1 / 2}\right) & =\sum_{m=-\infty}^{\infty} I_{m}(x a) K_{m}(x \rho) e^{i m \theta},
\end{aligned}
$$

and these series converge uniformly in $\theta$. Substituting these series in the above integrals we obtain for $\rho>a$

$$
\begin{aligned}
\frac{1}{2 \pi} \int_{-\infty}^{\infty} d \zeta \int_{0}^{2 \pi} d \sigma e^{i \beta \zeta}\left(e^{i k \Re} / \Re\right) \exp [i n(\sigma-\zeta / \alpha)] \\
=2 I_{n}\left(\left[(\beta-n / \alpha)^{2}-k^{2}\right]^{1 / 2} a\right) K_{n}\left(\left[(\beta-n / \alpha)^{2}-k^{2}\right]^{1 / 2} \rho\right) \\
\quad \text { if }(\beta-n / \alpha)^{2}>k^{2}, \\
=\pi i J_{n}\left(\left[k^{2}-(\beta-n / \alpha)^{2}\right]^{1 / 2} a\right) H_{n}^{(1)}\left(\left[k^{2}-(\beta-n / \alpha)^{2}\right]^{1 / 2} \rho\right) \\
\quad \text { if }(\beta-n / \alpha)^{2}<k^{2} .
\end{aligned}
$$


Now it can be shown [4, corollary 2.2] that $I_{n}(x) K_{n}(x)$ is a monotonic decreasing function of $x$ which assumes the value $1 /|2 n|$ at $x=0$. Since, in addition, $K_{n}(x)$ is monotonic decreasing in $x$, it follows that

$$
I_{n}\left(\Gamma_{n} a\right) K_{n}\left(\Gamma_{n} \rho\right)<1 /|2 n|
$$

for $\rho>a$. Combining this with the above result and the inequality (2.8), we obtain for fixed $\beta$ and fixed $\rho>a$

$$
\left|\int_{-L}^{L} d \zeta \int_{0}^{2 \pi} d \sigma e^{i \beta \zeta}\left(e^{i k R} / R\right) \exp [i n(\sigma-\zeta / \alpha)]\right|<\tilde{c} /|n|
$$

for sufficiently large $|n|$. It follows that $\lim _{L \rightarrow \infty} I(L, r)$ exists for $r<1$ and $\rho>a$; in fact

$$
\lim _{L \rightarrow \infty} I(L, r)=\sum_{n=-\infty}^{\infty} r^{|n|} 4 \pi I_{n}\left(\Gamma_{n} a\right) K_{n}\left(\Gamma_{n} \rho\right) e^{i n \varphi} .
$$

If for a particular $n, \Gamma_{n}$ is imaginary, then the corresponding term in (2.13) is to be replaced by $2 \pi^{2} i r^{|n|} J_{n}\left(\gamma_{n} \cdot a\right) H_{n}^{(1)}\left(\gamma_{n} \rho\right) e^{i n \zeta}$ where $\gamma_{n}=\left[k^{2}-(\beta-n / \alpha)^{2}\right]^{1 / 2}$. We shall in the future write the series as above and leave to the reader the interpretation for imaginary $\Gamma_{n}$.

In order to evaluate $\lim _{r \rightarrow 1-} \lim _{L \rightarrow \infty} I(L, r)$, we refer to an earlier paper [4, corollary 2.1]. It was there shown that for $n \geq 0$

$$
\frac{n}{v^{2}}\left[1+\frac{v^{2}}{n^{2}}\right]^{1 / 2}<\frac{-K_{n}^{\prime}(v)}{v K_{n}(v)}<\frac{n}{v^{2}}\left[1+\frac{v^{2}}{n(n-1)}\right]^{1 / 2} .
$$

Hence

$$
\frac{K_{n}^{\prime}(v)}{K_{n}(v)}<-\frac{n}{v}\left[1+\frac{v^{2}}{n^{2}}\right]^{1 / 2}<-\frac{n}{v}
$$

and

$$
K_{n}(v)<K_{n}\left(v_{0}\right)\left(v_{0} / v\right)^{n} \quad \text { for } \quad\left(v>v_{0}\right) .
$$

This coupled with the above mentioned fact that

$$
I_{n}(v) K_{n}(v) \leq 1 / 2 n
$$

gives

$$
I_{n}\left(\Gamma_{n} a\right) K_{n}\left(\Gamma_{n} \rho\right) \leq(a / \rho)^{n} / 2 n
$$

for $\rho>a$. Since $I_{-n}\left(\Gamma_{n} a\right) K_{-n}\left(\Gamma_{n} \rho\right)=I_{n}\left(\Gamma_{n} a\right) K_{n}\left(\Gamma_{n} \rho\right)$ the series (2.13) is majorized uniformly in $0 \leq r \leq 1$ by

$$
\sum_{n=-\infty}^{\infty}(a / \rho)^{|n|} /|2 n|
$$

Thus for $\rho>a$

$$
\lim _{r \rightarrow 1-} \lim _{L \rightarrow \infty} I(L, r)=\sum_{n=-\infty}^{\infty} 4 \pi e^{i n \varphi} I_{n}\left(\Gamma_{n} a\right) K_{n}\left(\Gamma_{n} \rho\right)
$$


Finally we shall establish (b), namely, that the $\lim _{r \rightarrow 1^{-}} I(L, r)$ exists uniformly in $L$. To this end, we define

$$
\begin{aligned}
& h_{\epsilon}(r, \sigma)=P(r, \sigma) \quad \text { for } \quad P(r, \sigma) \leq \epsilon, \\
& =\epsilon \quad \text { for } P(r, \sigma)>\epsilon, \\
& a(r, \epsilon)=1-\int_{0}^{2 \pi} d \sigma h_{\epsilon}(r, \sigma) \\
& f_{\epsilon}(r, \sigma)=h_{\epsilon}(r, \sigma) / a(r, \epsilon) \\
& g_{\epsilon}(r, \sigma)=\left[P(r, \sigma)-h_{\epsilon}(r, \sigma)\right] / a(r, \epsilon) .
\end{aligned}
$$

It is readily seen that

and

$$
\begin{gathered}
\int_{0}^{2 \pi} g_{\epsilon}(r, \sigma) d \sigma=1 \\
0<f_{\epsilon}(r, \sigma) \leq \epsilon / a(r, \epsilon) \leq \epsilon /(1-2 \pi \epsilon)
\end{gathered}
$$

$$
a(r, \epsilon) \rightarrow 1 \quad \text { as } \quad r \rightarrow 1^{-} .
$$

Now $f_{\epsilon}(r, \sigma)$ and $g_{\epsilon}(r, \sigma)$ are continuous functions of period $2 \pi$. Further $f_{\epsilon}(r, \sigma)$ is of total variation $2 \epsilon / a(r, \epsilon)$ over any interval of length $2 \pi$. On the other hand $g_{\epsilon}(r, \sigma)$ is a needle-like function, vanishing outside of an interval of length $\delta(\epsilon, r)$ about the origin, being otherwise positive valued and enclosing a unit area. It follows that any integral of the form $\int_{0}^{2 \pi} g_{\epsilon}(r, \sigma) w(\sigma) d \sigma$ differs from $w(0)$ by less than the oscillation of $w(\sigma)$ in the non-zero interval of $g_{\epsilon}(r, \sigma)$. It follows from known properties of $P(r, \sigma)$ that $\delta(\epsilon, r)$ approaches zero for each $\epsilon$ as $r \rightarrow 1^{-}$.

We write $I(L, r)$ as the sum of two integrals

$$
\begin{aligned}
I(L, r)=a(r, \epsilon) & \int_{-L}^{L} d \zeta \int_{0}^{2 \pi} d \sigma e^{i \beta \zeta}\left(e^{i k \Re} / \Re\right) f_{\epsilon}(r, \sigma-\zeta / \alpha) \\
& +a(r, \epsilon) \int_{-L}^{L} d \zeta \int_{0}^{2 \pi} d \sigma e^{i \beta \zeta}\left(e^{i k \Re} / \Re\right) g_{\epsilon}(r, \sigma-\zeta / \alpha)
\end{aligned}
$$

Expanding $f_{\epsilon}(r, \sigma)$ in a Fourier series, we obtain

where

$$
f_{\epsilon}(r, \sigma)=\sum_{n=-\infty}^{\infty} b_{n}(r, \epsilon) e^{i n \sigma},
$$

$$
\left|b_{n}(r, \epsilon)\right| \leq \operatorname{Var}\left(f_{\epsilon}\right) /|n| \leq 2 \epsilon /[|n| a(r, \epsilon)]
$$

Substituting this into the first of the integrals of (2.17) and interchanging the order of summation and integration (finite limits) gives

$$
\begin{aligned}
& a(r, \epsilon) \int_{-L}^{L} d \zeta \int_{0}^{2 \pi} d \sigma e^{i \beta \zeta}\left(e^{i k \Re} / \Re\right) f_{\epsilon}(r, \sigma-\zeta / \alpha) \\
& =\sum_{n=-\infty}^{\infty} a(r, \epsilon) b_{n}(r, \epsilon) \int_{-L}^{L} d \zeta \int_{0}^{2 \pi} d \sigma e^{i \beta \zeta}\left(e^{i k \Re} / \Re\right) \exp [i n(\sigma-\zeta / \alpha)]
\end{aligned}
$$


Finally if we combine this with the inequalities (2.12) and (2.18) we see that the series (2.19) is majorized by

$$
\epsilon \sum^{\prime} \tilde{c} / n^{2} \leq \epsilon C_{1}
$$

which converges to zero with $\epsilon$ uniformly in $L$ for fixed $\beta$ and fixed $\rho>a$.

The second integral in (2.17) approaches $\int_{-L}^{L} d \zeta e^{i \beta \zeta}\left(e^{i k R} / R\right)$ as $r \rightarrow 1^{-}$. Further, we note that the oscillation of $e^{i \beta \zeta}\left(e^{i \kappa \Re} / \Re\right)$ in the $\delta(\epsilon, r)$ interval about $\sigma=\zeta / \alpha$ is bounded by

$$
\left|\frac{\partial}{\partial \sigma}\left(e^{i k \Re} / \Re\right)\right| \delta(\epsilon, r) \leq C_{2} \delta(\epsilon, r) / \Re^{2}
$$

Hence

$$
\begin{aligned}
& \left|\int_{-L}^{L} d \zeta \int_{0}^{2 \pi} d \sigma e^{i \beta \zeta}\left(e^{i k \Re} / \Re\right) g_{\epsilon}(r, \sigma-\zeta / \alpha)-\int_{-L}^{L} d \zeta e^{i \beta \zeta}\left(e^{i k R} / R\right)\right| \\
& \quad \leq C_{2} \delta(\epsilon, r) \int_{-\infty}^{\infty} \Re^{-2} d \zeta=C_{3} \delta(\epsilon, r)
\end{aligned}
$$

uniformly in $L$. We have finally that

$$
\left|I(L, r) / a(r, \epsilon)-\lim _{r \rightarrow 1-} I(L, r)\right| \leq C_{1} \epsilon /(1-2 \pi \epsilon)+C_{3} \delta(\epsilon, r) .
$$

Since $\epsilon$ is arbitrary and $\delta(\epsilon, r)$ approaches zero as $r \rightarrow 1^{-}$, it follows that $\lim _{r \rightarrow 1-} I(L, r)$ exists uniformly in $L$. This concludes the proof of (b). Combining this with (a) it follows from a well known theorem on interchange of limits that

$$
\begin{aligned}
I & =\lim _{L \rightarrow \infty} \lim _{r \rightarrow 1-} I(L, r)=\lim _{r \rightarrow 1-} \lim _{L \rightarrow \infty} I(L, r) \\
& =\sum_{n=-\infty}^{\infty} 4 \pi e^{i n \varphi} I_{n}\left(\Gamma_{n} a\right) K_{n}\left(\Gamma_{n} \rho\right)
\end{aligned}
$$

for $\rho>a$.

It should be remarked that for a point inside of the helix, i.e., $\rho<a$, the vector potential components can be obtained by simply interchanging $a$ and $\rho$ since these two variables enter symmetrically in the integral $I$.

3. Electromagnetic field expressions. In the previous section it was shown that the integral $I$ converges for $\rho \neq a$. We now define

$$
I(\rho, \varphi)=\int_{-\infty}^{\infty} d \zeta e^{i \beta \zeta}\left(e^{i k R} / R\right)
$$

where $R=\left[\zeta^{2}+a^{2}+\rho^{2}-2 a \rho \cos (\varphi-\zeta / \alpha)\right]^{1 / 2}$ and $\beta$ is real. It remains to show that this integral converges for $\rho=a$ provided that $\varphi \neq 0$ (that is for a point not on the helix itself). This will be a by-product of our investigation into the derivatives of $I(\rho, \varphi)$ with respect to $\rho$ and $\varphi$.

For any pair of values $(\rho, \varphi)$ different from $(a, 0)$ there is a neighborhood of this point in which $R$ is bounded away from zero. Further $R$ is essentially equal to $|\zeta|$ for large $|\zeta|$. Hence for the points $(\rho, \varphi)$ of such neighborhood any integral majorized by 
$M / R^{\mu},(\mu>1)$, will converge uniformly. This is clearly the case for all derivatives of the integrand of $I(\rho, \varphi)$ with respect to $\rho$ and $\varphi$. It follows that

$$
\int_{\rho_{0}}^{\rho} d \rho \int_{-\infty}^{\infty} d \zeta \partial_{\rho}\left[e^{i \beta \zeta}\left(e^{i k R} / R\right)\right]=\int_{-\infty}^{\infty} d \zeta\left\{e^{i \beta \zeta}\left[\left(e^{i k R} / R\right)-\left(e^{i k R_{0}} / R_{0}\right)\right]\right\},
$$

where $R_{0}$ is obtained from $R$ by replacing $\rho$ by $\rho_{0}$.

Suppose now that $\rho_{0}=a$ whereas $\rho \neq a$. Then since $I(\rho, \varphi)$ converges, the righthand side of (3.2) can be written as the sum of two convergent integrals; hence $I(a, \varphi)$ converges for $\varphi \neq 0$. Whether $\rho_{0}=a$ or not, the integrand of the outer integral on the left-hand side of (3.2) is continuous. Hence

$$
\begin{aligned}
\partial_{\rho} I(\rho, \varphi) & =\partial_{\rho}\left[I(\rho, \varphi)-I\left(\rho_{0}, \varphi\right)\right]=\partial_{\rho}\left\{\int_{\rho_{0}}^{\rho} d \rho \int_{-\infty}^{\infty} d \zeta \partial_{\rho}\left[e^{i \beta \zeta}\left(e^{i k R} / R\right)\right]\right\} \\
& =\int_{-\infty}^{\infty} d \zeta \partial_{\rho}\left[e^{i \beta \zeta}\left(e^{i k R} / R\right)\right] .
\end{aligned}
$$

It is clear that a similar argument applies to all derivatives of $I(\rho, \varphi)$ with respect to $\rho$ and $\varphi$. Further we now know that $I$ converges for all points off of the wire. This is clearly independent of the coordinate system used to express the point. It follows therefore by precisely the same argument as above that the derivatives for the components of $\Pi$ with respect to $x, y$, and $z$ can be obtained by differentiating under the integral sign.

In order to evaluate the derivatives of $I(\rho, \varphi)$ for $\rho \neq a$, we recall the inequality (2.15) which shows that the series expansion for $I(\rho, \varphi)$ [see (2.20)] differentiated termwise with respect to $\varphi$, is majorized by $\sum(\rho / a)^{|n|}$ for $\rho<a$ and by $\sum(a / \rho)^{|n|}$ for $\rho>a$. It follows that $\partial_{\varphi} I(\rho, \varphi)$ can be obtained by termwise differentiation of the series expansion.

A similar result holds for $\partial_{\rho} I(\rho, \varphi)$. Here we make use of the inequality (2.14) for the case $\rho>a$. Thus

$$
\left|K_{n}^{\prime}(v)\right| \leq K_{n}(v) \frac{|n|}{v}\left[1+\frac{v^{2}}{n(n-1)}\right]^{1 / 2} .
$$

For $v \geq \Gamma_{n} a$, the functions $(|n| / v)\left[1+v^{2} / n(n-1)\right]^{1 / 2}$ are uniformly bounded by some positive number $M$. Hence

$$
\left|\Gamma_{n} I_{n}\left(\Gamma_{n} a\right) K_{n}^{\prime}\left(\Gamma_{n} \rho\right)\right| \leq \Gamma_{n} M I_{n}\left(\Gamma_{n} a\right) K_{n}\left(\Gamma_{n} \rho\right) \leq M_{1}(a / \rho)^{|n|},
$$

so that the series expansion for $I(\rho, \varphi)$ can be differentiated termwise with respect to $\rho$ in case $\rho>a$.

Finally $\rho<a$, we make use of the corresponding inequality for the $I_{n}$ function $(n \geq 0)$ (see [4, corollary 1.1])

$$
\frac{n}{v^{2}}\left[1+\frac{v^{2}}{n(n+1)}\right]^{1 / 2} \leq \frac{1}{v} \frac{I_{n}^{\prime}(v)}{I_{n}(v)} \leq \frac{n}{v^{2}}\left[1+\frac{v^{2}}{n^{2}}\right]^{1 / 2} .
$$

For $v \leq \Gamma_{n} a$, the functions $(n / v)\left[1+(v / n)^{2}\right]^{1 / 2}$ are uniformly bounded by $M^{\prime} / v$ for some positive number $M^{\prime}$. Hence

$$
\left|\Gamma_{n} I_{n}^{\prime}\left(\Gamma_{n} \rho\right) K_{n}\left(\Gamma_{n} a\right)\right| \leq\left(M_{1}^{\prime} / \rho\right)(\rho / a)^{|n|},
$$


so that again the series expansion can be differentiated termwise with respect to $\rho$ in the case $\rho<a$.

It can now be readily shown that the components of the vector potential (and hence the electromagnetic field components) satisfy the wave equation

$$
\Delta \Phi-\epsilon \mu \partial_{\imath} \Phi=0
$$

at all points $(x, y, z)$ not on the helix. In fact, since we can differentiate under the integral sign this amounts simply to showing that the quantity $e^{-i \omega t}\left(e^{i k r} / r\right)$ where $r=\left[(x-\xi)^{2}+(y-\eta)^{2}+(z-\zeta)^{2}\right]^{1 / 2}$ satisfies the wave equation (Note that $k^{2}=$ $\left.\epsilon \mu \omega^{2}\right)$.

We shall conclude this section by obtaining explicit expressions for the field components from the formulae (1.2) and (1.6). We first transform the integrals of (1.6) as in (1.7). Then we have

$$
\begin{aligned}
& \Pi_{\rho}=a F e^{i \beta z} \int_{-\infty}^{\infty} d \zeta e^{i \beta \zeta}\left(e^{i k r} / r\right) \sin (\varphi-z / \alpha-\zeta / \alpha), \\
& \Pi_{\varphi}=a F e^{i \beta z} \int_{-\infty}^{\infty} d \zeta e^{i \beta \zeta}\left(e^{i k r} / r\right) \cos (\varphi-z / \alpha-\zeta / \alpha), \\
& \Pi_{z}=\alpha F e^{i \beta z} \int_{-\infty}^{\infty} d \zeta e^{i \beta \zeta}\left(e^{i k r} / r\right),
\end{aligned}
$$

where

$$
r=\left[\zeta^{2}+a^{2}+\rho^{2}-2 a \rho \cos \left(\varphi-z_{/}^{\prime} \alpha-\zeta / \alpha\right)\right]^{1 / 2}
$$

We shall compute $E$ explicitly. Now

$$
\begin{aligned}
& \nabla \cdot \Pi=\frac{1}{\rho} \Pi_{\rho}+\partial_{\rho} \Pi_{\rho}+\frac{1}{\rho} \partial_{\varphi} \Pi_{\varphi}+\partial_{z} \Pi_{z} \\
& \partial_{\rho} \Pi_{\rho}=a F e^{i \beta z} \int_{-\infty}^{\infty} d \zeta e^{i \beta \zeta} r^{-1}[\rho-a \cos (\varphi-z / \alpha-\zeta / \alpha)] \sin (\varphi-z / \alpha-\zeta / \alpha) \partial_{r}\left(e^{i k r} / r\right) \\
& \partial_{\varphi} \Pi_{\varphi}=a F e^{i \beta z} \int_{-\infty}^{\infty} d \zeta e^{i \beta \zeta} r^{-1}\left[a \rho \sin (\varphi-z / \alpha-\zeta / \alpha) \cos (\varphi-z / \alpha-\zeta / \alpha) \partial_{r}\left(e^{i k r} / r\right)\right. \\
& \left.-\sin (\varphi-z / \alpha-\zeta / \alpha)\left(e^{i k r} / r\right)\right] \\
& \partial_{z} \Pi_{z}=i \beta \Pi_{z}+\alpha F e^{i \beta z} \int_{-\infty}^{\infty} d \zeta e^{i \beta \zeta}(\alpha r)^{-1}[-a \rho \sin (\varphi-z / \alpha-\zeta / \alpha)] \partial_{r}\left(e^{i k r} / r\right) .
\end{aligned}
$$

Combining the terms in $\nabla \cdot \Pi$ and making the appropriate cancellations gives

$$
\nabla \Pi=i \beta \Pi_{s}
$$

Since $E=\nabla(\nabla \cdot \Pi)+k^{2} \Pi$, we have for $\rho>a$ 


$$
\begin{aligned}
E_{\rho}= & i \beta \partial_{\rho} \mathrm{II}_{z}+k^{2} \Pi_{\rho} \\
= & 4 \pi i \beta \alpha F e^{i \beta z} \sum_{n=-\infty}^{\infty} \Gamma_{n} I_{n}\left(\Gamma_{n} a\right) K_{n}\left(\Gamma_{n} \rho\right) \exp [i n(\varphi-z / \alpha)] \\
& -i 2 \pi k^{2} a F e^{i \beta z} \sum_{n=-\infty}^{\infty}\left[I_{n-1}\left(\Gamma_{n} a\right) K_{n-1}\left(\Gamma_{n} \rho\right)\right. \\
& \left.-I_{n+1}\left(\Gamma_{n} a\right) K_{n+1}\left(\Gamma_{n} \rho\right)\right] \exp [i n(\varphi-z / \alpha)] \\
E_{\varphi}=\frac{i \beta}{\rho} \partial_{\varphi} \Pi_{z}+k^{2} \Pi_{\varphi} & \\
= & 4 \pi i \beta \alpha F \rho^{-1} e^{i \beta z} \sum_{n=-\infty}^{\infty} i n I_{n}\left(\Gamma_{n} a\right) K_{n}\left(\Gamma_{n} \rho\right) \exp [i n(\varphi-z / \alpha)] \\
& \quad+2 \pi k^{2} a F e^{i \beta z} \sum_{n=-\infty}^{\infty}\left[I_{n-1}\left(\Gamma_{n} a\right) K_{n-1}\left(\Gamma_{n} \rho\right)\right. \\
& \left.+I_{n+1}\left(\Gamma_{n} a\right) K_{n+1}\left(\Gamma_{n} \rho\right)\right] \exp [i n(\varphi-z / \alpha)] \\
E_{z}= & i \beta \partial_{z} \Pi_{z}+k^{2} \Pi_{z} \\
= & 4 \pi\left(k^{2}-\beta^{2}\right) \alpha F e^{i \beta z} \sum_{n=-\infty}^{\infty} I_{n}\left(\Gamma_{n} a\right) K_{n}\left(\Gamma_{n} \rho\right) \exp [i n(\varphi-z / \alpha)] \\
&
\end{aligned}
$$

The electric field components for $\rho<a$ are very similar. One can obtain the magnetic field components directly from

$$
H=-i \omega \epsilon \nabla \times \Pi \text {. }
$$

Since we shall have no occasion to use these in the present paper, we refrain from writing them out.

4. Determination of the propagation constant. In this section we shall determine the propagation constant $\beta$ so that the electric field along the helix is "essentially" zero. Since we assume the helix to be a perfect conductor this is the proper boundary condition for our problem. The electric field in the helical direction at any point $(\rho, \varphi, z)$ is

$$
E_{h}=\left(\alpha /\left[a^{2}+\alpha^{2}\right]^{1 / 2}\right) E_{z}+\left(a /\left[a^{2}+\alpha^{2}\right]^{1 / 2}\right) E_{\varphi} .
$$

In order to approach the helix radially, we set $\varphi=z / a$ in (4.1) and try to determine $\beta$ so that $\lim _{\rho \rightarrow a} E_{h}=0$. We will suppose that $\rho>a$; the case $\rho<a$ is entirely similar.

Because of the singular nature of the field about an infinitely thin conductor we cannot actually choose $\beta$ so that $\lim _{\rho \rightarrow a} E_{h}=0$. Since $E$ becomes infinite as $\rho \rightarrow a$, the best we can do is to determine the proper phase velocity, $\beta_{0}$, so that

$$
\lim _{\rho \rightarrow a} \frac{E_{h}\left(\beta_{0}\right)}{E_{h}\left(|\beta| \neq\left|\beta_{0}\right|\right)}=0 .
$$


For $\varphi=z / \alpha$ and $\rho>a, E_{h}$ can be expressed as

$$
\begin{aligned}
{\left[a^{2}+\alpha^{2}\right]^{1 / 2} E_{h}=4 \pi \alpha^{2}\left(k^{2}-\beta^{2}\right) F e^{i \beta z} \sum_{n=-\infty}^{\infty} I_{n}\left(\Gamma_{n} a\right) K_{n}\left(\Gamma_{n} \rho\right) } \\
+2 \pi a^{2} k^{2} F e^{i \beta z} \sum_{n=-\infty}^{\infty}\left[I_{n-1}\left(\Gamma_{n} a\right) K_{n-1}\left(\Gamma_{n} \rho\right)+I_{n+1}\left(\Gamma_{n} a\right) K_{n+1}\left(\Gamma_{n} \rho\right)\right] \\
-4 \pi i \beta \alpha\left(1-a_{/} \rho\right) F e^{i \beta z} \sum_{n=-\infty}^{\infty} i n I_{n}\left(\Gamma_{n} a\right) K_{n}\left(\Gamma_{n} \rho\right) .
\end{aligned}
$$

We shall first prove that the last term in (4.3) approaches zero as $\rho \rightarrow a$. In its original integral form this term becomes

$$
J(\rho)=\left(1-a_{/} \rho\right)(a \rho)^{-1} \partial_{\varphi}[I(\rho, \varphi)]_{\varphi=0}=(1-a / \rho) \int_{-\infty}^{\infty} e^{i \beta \zeta} R^{-1} \sin \zeta \partial_{R}\left(e^{i k R} / R\right) d \zeta
$$

aside from a factor independent of $\rho$. Here

$$
R=\left[\zeta^{2}+a^{2}+\rho^{2}-2 a \rho \cos \zeta / \alpha\right]^{1 / 2}
$$

is an even function of $\zeta$. The $e^{i \beta \zeta}$ can be expanded as

$$
e^{i \beta \zeta}=\cos \beta \zeta+i \sin \beta \zeta .
$$

Since $\left[\cos \beta \zeta R^{-1} \sin \zeta \partial_{R}\left(e^{i k R} / R\right)\right]$ is odd, its integral vanishes.

Hence

$$
J(\rho)=(1-a / \rho) \int_{-\infty}^{\infty} R^{-1} \sin \beta \zeta \sin \zeta \partial_{R}\left(e^{i k R} / R\right) d \zeta .
$$

We note that

$$
|\zeta| \leq\left[\zeta^{2}+(a-\rho)^{2}\right]^{1 / 2} \leq R
$$

It follows that there exist constants $c_{1}$ and $c_{2}$ such that

$$
\begin{aligned}
& \left|\partial_{R}\left(e^{i k R} / R\right)\right| \leq c_{1} /|\zeta| \quad \text { for } \quad|\zeta|>1 \text {, } \\
& \leq c_{2}\left[\zeta^{2}+(a-\rho)^{2}\right] \quad \text { for } \quad|\zeta| \leq 1 \quad \text { and } \quad|\rho-a|<1 .
\end{aligned}
$$

Therefore we have

$$
\begin{array}{r}
|J(\rho)| \leq(1-a / \rho)\left\{2 \int_{1}^{\infty} d \zeta c_{1} / \zeta^{2}+2 \int_{0}^{1} d \zeta \beta c_{2} \zeta^{2}\left[\zeta^{2}+(a-\rho)^{2}\right]^{-3 / 2}\right\} \\
=(1-a / \rho)\left\{2 c_{1}+2 \beta c_{2}\left[\log \left\{\left(1+\left[1+(a-\rho)^{2}\right]^{1 / 2}\right) /(\rho-a)\right\}\right.\right. \\
\left.\left.-\left\{1+(a-\rho)^{2}\right\}^{-1 / 2}\right]\right\}
\end{array}
$$

which approaches zero as $\rho \rightarrow a^{+}$.

In determining the limit of $E_{h}$ as $\rho \rightarrow a^{+}$, we can therefore omit the last term in (4.3). We denote by $E_{h}^{\prime}$ the thus abbreviated $E_{h}$. We now define the function

$$
\Omega(\beta, \rho)=\sum_{n=-\infty}^{\infty} I_{n}\left(\Gamma_{n} a\right) K_{n}\left(\Gamma_{n} \rho\right) .
$$


Then for $\varphi=z / \alpha$ and $\rho>a$, we have

$$
\begin{aligned}
E_{h}^{\prime}=\frac{4 \pi F}{\left[a^{2}+\alpha^{2}\right]^{1 / 2}} e^{i \beta_{z}}\left[\alpha^{2}\left(k^{2}-\beta^{2}\right) \Omega(\beta, \rho)\right. \\
\left.\quad+\left(a^{2} k^{2} / 2\right)\{\Omega(\beta-1 / \alpha, \rho)+\Omega(\beta+1 / \alpha, \rho)\}\right] .
\end{aligned}
$$

It turns out that

$$
\lim _{\rho \rightarrow a^{+}} \Omega(\beta, \rho)=\infty,
$$

so that we can hardly expect to obtain a $\beta$ for which $\lim _{\rho \rightarrow a^{+}} E_{h}=0$. However we shall also prove that

Hence

$$
\lim _{\rho \rightarrow a^{+}} \Omega\left(\beta_{1}, \rho\right) / \Omega\left(\beta_{2}, \rho\right)=1 .
$$

$$
\lim _{\rho \rightarrow a^{+}} \frac{E_{h}(\beta)}{E_{h}\left(\beta_{1}\right)}=\frac{\alpha^{2}\left(k^{2}-\beta^{2}\right)+a^{2} k^{2}}{\alpha^{2}\left(k^{2}-\beta_{1}^{2}\right)+a^{2} k^{2}}
$$

and for $\beta_{1}^{2} \neq \beta^{2}$. This vanishes if and only if

$$
\alpha^{2}\left(k^{2}-\beta^{2}\right)+a^{2} k^{2}=0 .
$$

In other words the correct propagation constant $\beta_{0}$ is simply

$$
\beta_{0}= \pm k\left[a^{2}+\alpha^{2}\right]^{1 / 2} \alpha^{-1} .
$$

We now prove (4.6). In order to do this we make use of the inequalities (2.14) and (3.3), from which it follows that

$$
-\frac{K_{n}^{\prime}(v)}{v K_{n}(v)}-\frac{I_{n}^{\prime}(v)}{v I_{n}(v)}<\frac{n}{v^{2}}\left\{\left[1+\frac{v^{2}}{n(n-1)}\right]^{1 / 2}-\left[1+\frac{v^{2}}{n(n+1)}\right]^{1 / 2}\right\} .
$$

The expression on the right is monotonic decreasing achieving its maximum of $1 /\left(n^{2}-1\right)$ at $v=0$. Hence

$$
\frac{\left(I_{n}(v) K_{n}(v)\right)^{\prime}}{I_{n}(v) K_{n}(v)}>-v /\left(n^{2}-1\right)
$$

and since $I_{n}(0) K_{n}(0)=1 /(2 n)$, we obtain

$$
(2 n)^{-1} \geq I_{n}(v) K_{n}(v) \geq(2 n)^{-1} \exp \left\{-v^{2} /\left[2\left(n^{2}-1\right)\right]\right\} .
$$

Finally for sufficiently large $|n|$,

and hence

$$
\Gamma_{n}=\left[(\beta-n / \alpha)^{2}-k^{2}\right]^{1 / 2}>c|n|
$$

$$
I_{n}\left(\Gamma_{n} a\right) K_{n}\left(\Gamma_{n} a\right) \geq|2 n|^{-1} \exp \left(-c^{2}\right) .
$$

Since $\Omega(\beta, \rho)$ consists of positive continuous terms, monotonically increasing as $\rho \rightarrow a^{+}$, we have

$$
\lim _{\rho \rightarrow a^{+}} \Omega(\beta, \rho)=\Omega(\beta, a) \gg(1 / 2) \exp \left(-c^{2}\right) \sum_{n=-\infty}^{\infty},|n|^{-1},
$$

which diverges. 
We shall next establish (4.7) also by means of the inequalities (2.14) and (3.3). Now clearly

$$
\begin{aligned}
\frac{\partial_{v}\left[I_{n}(v a) K_{n}(v \rho)\right]}{I_{n}(v a) K_{n}(v \rho)} & =a \frac{I_{n}^{\prime}(v a)}{I_{n}(v a)}+\rho \frac{K_{n}^{\prime}(v \rho)}{K_{n}(v \rho)} \\
& =v\left\{a^{2}\left[\frac{I_{n}^{\prime}(v a)}{v a I_{n}(v a)}\right]-\rho^{2}\left[\frac{-K_{n}^{\prime}(v)}{v K_{n}(v)}\right]\right\} .
\end{aligned}
$$

It follows from (2.14) and (3.3) that for $n \geq 1$ and $v>0$

$$
\begin{aligned}
\frac{\partial_{v}\left[I_{n}(v a) K_{n}(v \rho)\right]}{I_{n}(v a) K_{n}(v \rho)} & \leq v\left\{a^{2} \frac{n}{(v a)^{2}}\left[1+\frac{(v a)^{2}}{n^{2}}\right]^{1 / 2}-\rho^{2} \frac{n}{(v \rho)^{2}}\left[1+\frac{(v \rho)^{2}}{n^{2}}\right]^{1 / 2}\right\} \\
& \leq \frac{n}{v}\left\{\left[1+\frac{(v a)^{2}}{n^{2}}\right]^{1 / 2}-\left[1+\frac{(v \rho)^{2}}{n^{2}}\right]^{1 / 2}\right\}<0
\end{aligned}
$$

for $\rho>a$. Likewise $(n \geq 1$ and $v \geq 0)$

$$
\frac{\partial_{v}\left[I_{n}(v a) K_{n}(v \rho)\right]}{I_{n}(v a) K_{n}(v \rho)} \geq v\left\{\frac{n}{v^{2}}\left[1+\frac{(v a)^{2}}{n(n+1)}\right]^{1 / 2}-\frac{n}{v^{2}}\left[1+\frac{(v \rho)^{2}}{n(n-1)}\right]^{1 / 2}\right\} .
$$

It is easily shown that the function within the brackets is negative monotonic increasing for $\rho>a$, achieving a minimum of

$$
\left(-\frac{n\left(\rho^{2}-a^{2}\right)+\left(\rho^{2}+a^{2}\right)}{n^{2}-1}\right) \quad \text { at } \quad v=0 .
$$

Hence

$$
\frac{\partial_{v}\left[I_{n}(v a) K_{n}(v \rho)\right]}{I_{n}(v a) K_{n}(v \rho)} \geq-\omega_{n} v
$$

where

$$
\omega_{n}=\frac{n\left(\rho^{2}-a^{2}\right)+\left(\rho^{2}+a^{2}\right)}{n^{2}-1} .
$$

If we now integrate the above logarithmic derivative from $v>0$ to $v+\Delta>0$ we obtain for $n \geq 1$

$\exp \left(-\frac{\omega_{n}}{2}\left[(v+\Delta)^{2}-v^{2}\right]\right) \leq \frac{I_{n}[(v+\Delta) a] K_{n}[(v+\Delta) \rho]}{I_{n}(v a) K_{n}(v \rho)} \leq 1 \quad$ for $\quad \Delta \geq 0$

and

$1 \leq \frac{I_{n}[(v+\Delta) a] K_{n}[(v+\Delta) \rho]}{I_{n}(v a) K_{n}(v \rho)} \leq \exp \left(-\frac{\omega_{n}}{2}\left[(v+\Delta)^{2}-v^{2}\right]\right) \quad$ for $\quad \Delta \leq 0$.

Finally because $I_{-n}(v a) K_{-n}(v \rho)=I_{n}(v a) K_{n}(v \rho)$, the above inequalities hold for $n \leq-1$ if we replace $n$ in $\omega_{n}$ by $|n|$. We can combine (4.9a) and (4.9b) into

$$
e^{-\epsilon(\rho, n)} \leq \frac{I_{n}[(v+\Delta) a] K_{n}[(v+\Delta) \rho]}{I_{n}(v a) K_{n}(v \rho)} \leq e^{\epsilon(\rho, n)}
$$


for all $|n|>1, v>0,(v+\Delta)>0$, and $\rho>a$, where

$$
\epsilon(\rho, n)=\frac{|n|\left(\rho^{2}-a^{2}\right)+\rho^{2}+a^{2}}{n^{2}-1}\left|(v+\Delta)^{2}-v^{2}\right| .
$$

It will be seen from the expression for $\Omega(\beta, \rho)$ that the appropriate values for $v$ and $(v+\Delta)$ in $I_{n}(v a) K_{n}(v \rho)$ are

$$
v_{n}=\left[\left(\beta_{1}-\frac{n}{\alpha}\right)^{2}-k^{2}\right]^{1 / 2}=\Gamma_{n}^{(1)}
$$

and

$$
(v+\Delta)_{n}=\left[\left(\beta_{2}-\frac{n}{\alpha}\right)^{2}-k^{2}\right]^{1 / 2}=\Gamma_{n}^{(2)} .
$$

Hence

$$
(v+\Delta)_{n}^{2}-v_{n}^{2}=\beta_{2}^{2}-\beta_{1}^{2}-2 n\left(\beta_{2}-\beta_{1}\right) / \alpha .
$$

It is clear that $\epsilon(\rho, n)$ is the sum of two parts one of which is of the order of $(\rho-a)$ and the other of the order of $1 /(|n|)$. Hence given $\beta_{1}, \beta_{2}$, and $\eta>0$, there exists an $N$ and a $\delta>0$ such that for $|n| \geq N$ and $0<\rho-a<\delta$

$$
\epsilon(\rho, n)<\eta \text {. }
$$

The finite sums $\sum_{-N}^{N} I_{n}\left(\Gamma_{n}^{(i)} a\right) K_{n}\left(\Gamma_{n}^{(i)} \rho\right)$, remain uniformly bounded as $\rho \rightarrow a^{+}$. Hence

$$
\begin{aligned}
1-\frac{\Omega\left(\beta_{1}, \rho\right)}{\Omega\left(\beta_{2}, \rho\right)} & =\frac{\sum_{-N}^{N}\{\}+\sum_{-\infty}^{-N}\{\}+\sum_{N}^{\infty}\{\}}{\Omega\left(\beta_{2}, \rho\right)} \\
& =\frac{\sum_{-N}^{N}\{\}}{\Omega\left(\beta_{2}, \rho\right)}+\frac{\sum_{-\infty}^{-N}\{\}+\sum_{N}^{\infty}\{\}}{\Omega\left(\beta_{2}, \rho\right)},
\end{aligned}
$$

where \{\} stands for the running term

$$
\left\{I_{n}\left(\Gamma_{n}^{(2)} a\right) K_{n}\left(\Gamma_{n}^{(2)} \rho\right)-I_{n}\left(\Gamma_{n}^{(1)} a\right) K_{n}\left(\Gamma_{n}^{(1)} \rho\right)\right\} ;
$$

thus

$$
\left|1-\frac{\Omega\left(\beta_{1}, \rho\right)}{\Omega\left(\beta_{2}, \rho\right)}\right| \leq \frac{\left|\sum_{-N}^{N}\{\}\right|}{\Omega_{2}(\beta, \rho)}+\frac{\left(e^{\eta}-e^{-\eta}\right)\left[\Omega\left(\beta_{2}, \rho\right)-\sum_{-N}^{N} I_{n}\left(\Gamma_{n}^{(2)} a\right) K_{n}\left(\Gamma_{n}^{(2)} \rho\right)\right]}{\Omega\left(\beta_{2}, \rho\right)} .
$$

It follows that

$$
\varlimsup_{\rho \rightarrow a^{+}}\left|1-\frac{\Omega\left(\beta_{1}, \rho\right)}{\Omega\left(\beta_{2}, \rho\right)}\right| \leq 0+e^{\eta}-e^{-\eta},
$$

and since $\eta$ is arbitrarily small, we have

$$
\lim _{\rho \rightarrow a^{+}} \frac{\Omega\left(\beta_{1}, \rho\right)}{\Omega\left(\beta_{2}, \rho\right)}=1 .
$$

5. Conclusion. The net result of our investigation has been to obtain expressions for the electromagnetic field which satisfy the Maxwell equations away from the helix and 
which satisfy the appropriate boundary conditions along the helix. The propagation constant in the axial direction was found (4.8) to be

$$
\beta_{0}= \pm k\left[a^{2}+\alpha^{2}\right]^{1 / 2} \alpha^{-1}
$$

which corresponds to precisely the phase velocity one would obtain if the wave traveled along the helix itself with the free space velocity of propagation.

In case

$$
\left(\beta_{0}-n / \alpha\right)^{2}>k^{2}
$$

for all $n(n=0, \pm 1, \pm 2, \cdots)$ none of the terms in the expansion of the field equations are unmodified Hankel functions. As a consequence there is no net radial flow of energy. On the other hand for a given helix there is a series of frequency bands for which the inequality (5.2) is not satisfied for all $n$; in this case a finite number of Hankel function terms do appear in the field expressions. It can be shown that these terms do cause a net radial flow of energy. One might expect this phenomena to be reflected in the propagation constant $\beta_{0}$; that is, one would expect $\beta_{0}$ to be complex. More precisely, unless there is a source of energy within the wire itself, one would expect

$$
\operatorname{Im}\left(\beta_{0}\right)=\frac{1}{2} \frac{\text { Energy radiated per unit distance in the } z \text {-direction }}{\text { Energy flow in the } z \text {-direction }} .
$$

However the computed value of $\beta_{0}$ (5.1) is actually consistent with (5.3) for it can be shown that whereas the energy radiated per unit distance in the $z$-direction is finite, the energy flow in the $z$-direction is infinite. As a matter of fact not only is the total contribution of the set of terms satisfying (5.2) infinite, but the contribution of each of the radiating terms to the energy flow in the $z$-direction is itself infinite.

In spite of the physical reasonableness of our result, there is, in the case of the radiating helix, reason to be dissatisfied with it. If (5.2) is satisfied for all $n$, the expansion which we have obtained can be used for helices with conductors of finite thickness. One could, for instance, replace the delta function of section 2 by a finite needle function (say $P\left(r_{0}, \sigma\right)$ with fixed $\left.r_{0}<1\right)$. The helix would then be constructed from infinitely thin tape. For instance $\Pi_{z}$ would be of the type $(\rho>a)$

$$
\Pi_{z}=\alpha F^{\prime} e^{i \beta z} \sum_{n=-\infty}^{\infty} a_{n} I_{n}\left(\Gamma_{n} a\right) K_{n}\left(\Gamma_{n} \rho\right) \exp [\operatorname{in}(\varphi-z / \alpha)]
$$

where the $\left(a_{n}\right)$ are Fourier coefficients of the needle function. Repeating the above development one would obtain a real $\beta_{0}$ for the $a_{n}$ 's sufficiently close to 1 . However, in the radiating case, one would not be able to obtain a solution in this way. In this case, no real $\beta$ would allow the boundary conditions to be satisfied whereas the entire development (starting with the vector potential integrals) is meaningless for complex $\beta$. This is also reflected by the fact that a complex $\beta$ which satisfies the boundary conditions would make the arguments of all of the Hankel functions complex so that the field would decay exponentially. As a consequence we would have no radiating terms which would be contrary to (5.3). Thus in case (5.2) is not satisfied by all $n$, one would expect a real helical wire to radiate. However one would not expect the field expressions for finite conductors to have expansions of the type which we have obtained for this case. 


\section{REFERENCES}

1. Franz Ollendorff, Die Grundlagen der Hochfrequenztechnik, Springer, Berlin, 1926, pp. 79-87.

2. J. R. Pierce, Theory of the beam-type traveling-wave tube, Proceedings of the Institute of Radio Engineers 35, 111-123 (1947).

3. R. S. Phillips and Henry Malin, A helical wave guide, New York University Mathematical Research Group, Report No. 170-3, Army Air Forces, Watson Laboratories, 1947.

4. R. S. Phillips and Henry Malin, Bessel function approximations, to be published in the Amer. J. Math.

5. S. Ramo and J. R. Whinnery, Fields and waves in modern radio, Wiley and Sons, New York, 1944.

6. G. N. Watson, Theory of Bessel functions, MacMillan, New York, 1944.

7. A. Zygmund, Trigonometrical series, Monografje Matematyczne, Warsaw, 1935. 